\title{
An RF Sensor for Gauging Screen-Channel Liquid Acquisition Devices for Cryogenic Propellants
}

\author{
Gregory A. Zimmerli* \\ National Aeronautics and Space Administration Glenn Research Center, Cleveland, Ohio, 44135 \\ Scott Metzger ${ }^{\dagger}$ \\ Vantage Partners, LLC, Cleveland, Ohio, 44135 \\ Marius Asipauskas \\ National Center for Space Exploration Research, Cleveland, Ohio, 44135
}

\begin{abstract}
A key requirement of a low-gravity screen-channel liquid acquisition device (LAD) is the need to retain $100 \%$ liquid in the channel in response to propellant outflow and spacecraft maneuvers. The point at which a screen-channel LAD ingests vapor is known as breakdown, and can be measured several different ways such as: visual observation of bubbles in the LAD channel outflow; a sudden change in pressure drop between the propellant tank and LAD sump outlet; or, an indication by wet-dry sensors placed in the LAD channel or outflow stream. Here we describe a new type of sensor for gauging a screen-channel LAD, the Radio Frequency Mass Gauge (RFMG). The RFMG measures the natural electromagnetic modes of the screen-channel LAD, which is very similar to an RF waveguide, to determine the amount of propellant in the channel. By monitoring several of the RF modes, we show that the RFMG acts as a global sensor of the LAD channel propellant fill level, and enables detection of LAD breakdown even in the absence of outflow. This paper presents the theory behind the RFMG-LAD sensor, measurements and simulations of the RF modes of a LAD channel, and RFMG detection of LAD breakdown in a channel using a simulant fluid during inverted outflow and long-term stability tests.
\end{abstract}

$\begin{array}{ll}\text { LAD } & =\text { Liquid Acquisition Device } \\ \text { NRC } & =\text { National Research Council } \\ \text { PMD } & =\text { Propellant Management Device }\end{array}$
Abbreviations

$$
\begin{array}{ll}
\text { RF } & =\text { Radio Frequency } \\
\text { RFMG } & =\text { Radio Frequency Mass Gauge } \\
\text { TRL } & =\text { Technology Readiness Level }
\end{array}
$$

\section{Introduction}

N 2010, NASA released a draft of its Space Technology Roadmap to help guide the dev
technologies ${ }^{1}$. The roadmap contains fourteen different technical areas including In-S
The National Research Council provided an independent review of the draft roadmap,
study were published in a 2012 report which also identified high-priority technologies
Within the In-Space Propulsion Systems technical area, cryogenic propellant storage
technologies identified by the NRC as a high-priority. In order to enable the transfer
gravity, either to another tank such as a depot or a propulsion feed-system, a low-grav
(LAD) is needed to acquire liquid from practically anywhere inside the tank and mak
outflow system. One type of LAD which operates passively and is based on capillary
${ }^{*}$ Research Aerospace Engineer, Propulsion and Propellants, Greg.Zimmerli@nasa.gov
${ }^{\dagger}$ Electrical Engineer, Scott.L.Metzger@nasa.gov
${ }^{*}$ Research Engineer, Marius.Asipauskas-1@nasa.gov

American Institute of Aeronautics and Astronautics 
screen-channel type LAD. Although LAD's have been used successfully for storable propellants ${ }^{3}$ and superfluid helium ${ }^{4}$ in low-gravity, they have not been tested in low-gravity with a cryogenic propellant. Each type of LAD has some advantage and disadvantage which needs to be assessed based on the system requirements. When a moderate rate of propellant outflow is desired even during periods of adverse accelerations, the screen-channel LAD is generally preferred ${ }^{5}$.

A key requirement for the LAD system is to acquire vapor-free liquid and make it available for transfer to the propellant feed system ${ }^{6}$. The ingestion of vapor into the feed system can potentially lead to problems such as inefficient propellant transfer, pump damage (if a pump is used in the feed system), or engine damage if the feed system is delivering propellant to an engine. Thus, it may be advantageous to have a sensor which can detect vapor in the LAD system before it reaches the feed system. Although not used in a LAD, a state-of-the-art sensor of this type is an engine cut-off sensor, which senses a wet-dry transition to detect vapor before it reaches the tank outlet. The space shuttle liquid hydrogen external tank, for example, used wet-dry sensors as engine cut-off sensors. Although a few such sensors can be used for a settled-liquid application, the use of point sensors for a low-gravity application may not reliably detect ingestion of vapor into the LAD or feed system unless, perhaps, a large number of sensors are used.

In this paper we describe a new type of sensor that can be used in a low-gravity propellant management device (PMD) such as a screen-channel LAD or screened-sump to detect vapor ingestion before it reaches the tank outlet or feed system. The sensor is based on the same operating principle as the RFMG ${ }^{7,8}$. The RFMG measures the natural electromagnetic modes of the LAD, which are affected by the presence of the dielectric liquid. By measuring a change in the frequency of the modes, it is possible to detect vapor bubbles inside the LAD. This paper presents the principle of operation of the RFMG sensor, measurement and simulations of a screen-channel LAD RF modes, and RFMG detection of LAD breakdown in a screen channel using $3 \mathrm{M}^{\mathrm{TM}}$ Fluorinert ${ }^{\mathrm{TM}}$ FC-77 as a simulant fluid ${ }^{9}$.

\section{Principle of Operation}

A typical screen channel LAD is comprised of a rectangular channel, three sides of which are solid metal, and the fourth side being the porous screen. Figure 1 shows the LAD channel used in the tests reported here; both the channel and screen are constructed from stainless steel. When incorporated into a flight system, the screen side of the channel faces the tank wall, but mounted slightly away from the wall to allow liquid to pass into the channel, and the LAD channel is manufactured to conform to the tank wall curvature. Several LAD channels may be mounted in a tank, connecting together at a sump outlet. The screen inhibits the passage of vapor from the tank ullage into the $\mathrm{LAD}$, thus providing a continuous supply of bubble-free liquid to the sump. The fine mesh screen inhibits the

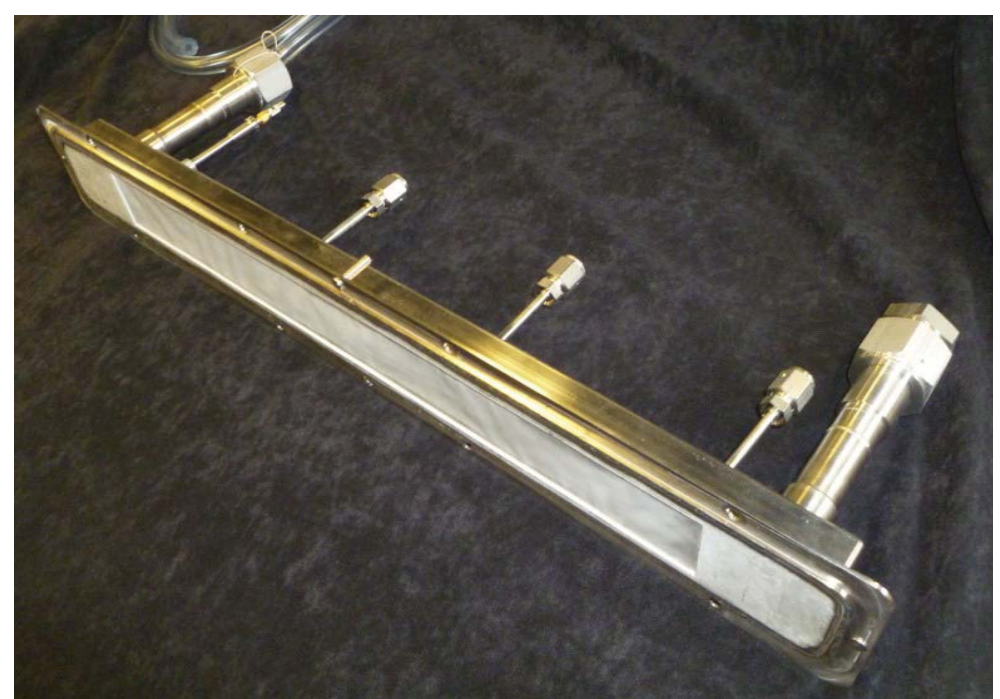

Figure 1. Photo of the screen-channel LAD used in these tests. passage of vapor through capillary action of the liquid along the screen mesh. When fully wetted by the liquid, the screen mesh can reject the passage of vapor across the screen as long as the pressure difference between the tank ullage and LAD channel is less than the bubble-point pressure, $P_{B P}$, given by

$$
P_{B P}=\frac{4 \gamma}{D_{P}}
$$

where $\gamma$ is the liquid-vapor surface tension of the liquid, and $D_{P}$ is the effective pore diameter of the screen mesh. For the $325 \times 2300$ Dutch twill screen used in this LAD channel, the effective pore diameter calculated from bubble-point measurements is 14.0 microns ${ }^{10}$. In order to characterize the effective pore diameter of a screen, the bubble-point pressure is often measured using isopropyl alcohol, and (1) is then used to calculate the effective pore diameter. In $1 \mathrm{~g}$ ground tests 
where the LAD is oriented vertically, the LAD channel can hold a column of liquid of maximum height $h_{\max }$ determined by equating the liquid column head pressure with the bubble-point pressure:

$$
h_{\max }=\frac{4 \gamma}{\rho g D_{P}}
$$

where $\rho$ is the density of the fluid and $g$ the acceleration due to Earth's gravity. Equation (2) is strictly valid for a static liquid (no flow) since it neglects the flow-induced pressure drop across the screen. At low flow rates, however, (2) is a very good approximation.

The liquid level in the LAD was monitored in these tests using the same principle as RFMG, albeit at higher microwave frequencies. In operation, the RFMG detects the electromagnetic eigenmodes, or natural resonant frequencies, of a tank (or LAD channel in this case) containing a dielectric fluid such as a cryogenic propellant. The essential hardware components, shown in Figure 2, consist of the LAD channel, an internally mounted antenna probe, an RF reflectometer that measures the reflected power from the antenna probe, and a computer. At a resonant frequency there is a drop in the reflected RF power, and these inverted peaks in the reflected power spectrum, shown in Figure 3 as the return loss or $\mathrm{S}_{11}$ spectrum, are identified as the LAD channel eigenmode frequencies using a peak-detection software algorithm. This eigenmode frequency information is passed to a pattern matching algorithm, which compares the measured eigenmode frequencies with a database of simulated eigenmode frequencies at various fill levels and liquid configurations. The database of simulated eigenmode frequencies is created in advance using electromagnetic simulation software to calculate the LAD channel eigenmodes at various fill levels for a vertically oriented channel. A best match between the simulated and measured mode frequency values occurs at some fill level, which is then reported as the gauged fill level.

The natural electromagnetic mode frequencies of a rectangular channel closed at both ends are given by

$$
f_{m n l}=\frac{c}{2} \sqrt{\left(\frac{m}{a}\right)^{2}+\left(\frac{n}{b}\right)^{2}+\left(\frac{l}{L}\right)^{2}}
$$

where $c$ is the speed of light, $m=0,1,2, . ., n=0,1,2, \ldots$ but $(m, n) \neq(0,0), l=1,2,3, \ldots$ are the mode indices, and $a$, $b, L$ are the height, width, and length of the channel. The LAD channel used in these tests had dimensions $a=2.5$ $\mathrm{cm}, b=5.1 \mathrm{~cm}$, and $L=61.0 \mathrm{~cm}$. For a channel length $L>>b>a$, the lowest modes of the channel are given by $m=$ $0, n=1$, and $l=1,2, \ldots$ Equation (3) provides an excellent estimate of the empty LAD channel mode frequencies, but for the highest accuracy it is necessary to include other details of the LAD channel, such as fluid line ports and the antenna in a computer finite element model. Our simplified model of the LAD channel included the two larger fluid line ports ( $2.5 \mathrm{~cm}$ diameter) and the $1.2 \mathrm{~cm}$ long antenna. The model was created in $\mathrm{COMSOL}^{11}$, and the RF

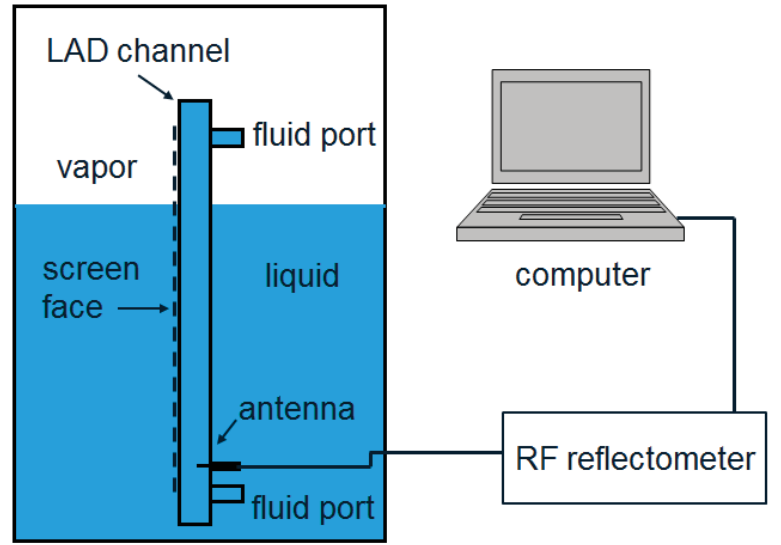

Figure 2. Schematic of the test setup used for RF measurements of liquid quantity in the LAD channel.

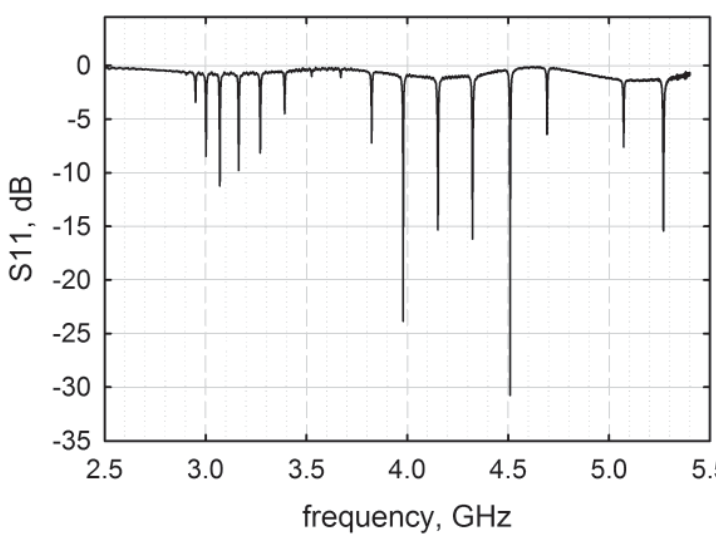

Figure 3. RF spectrum of the empty LAD channel. 
Module was used to compute the LAD mode frequencies as a function of liquid level using a measured dielectric constant of $\varepsilon=1.827$ for the FC-77 liquid. Figure 4 shows the calculated mode frequencies as a function of liquid fill level in the vertical LAD channel. The database of simulated mode frequencies is used in the gauging algorithm to determine the liquid level in the LAD channel.

The lowest mode was not detected in the empty LAD spectrum, but was detected at some fill levels. When a mode's electric field amplitude is near a minimum at the antenna location, the coupling to the mode is poor, resulting in poor coupling or no coupling at all to the mode.

\section{Test Setup and Procedures}

The LAD channel was mounted inside a 108.1 L capacity tank which was attached to a rotatable rig. The rotation rig was useful for orienting the LAD channel in such a way as to enable removal of air bubbles during the tank and LAD channel fill procedure. The long axis of the LAD channel was aligned with the long axis of the tank, and was kept in the vertical position during testing except when the tank and LAD were being filled with liquid.

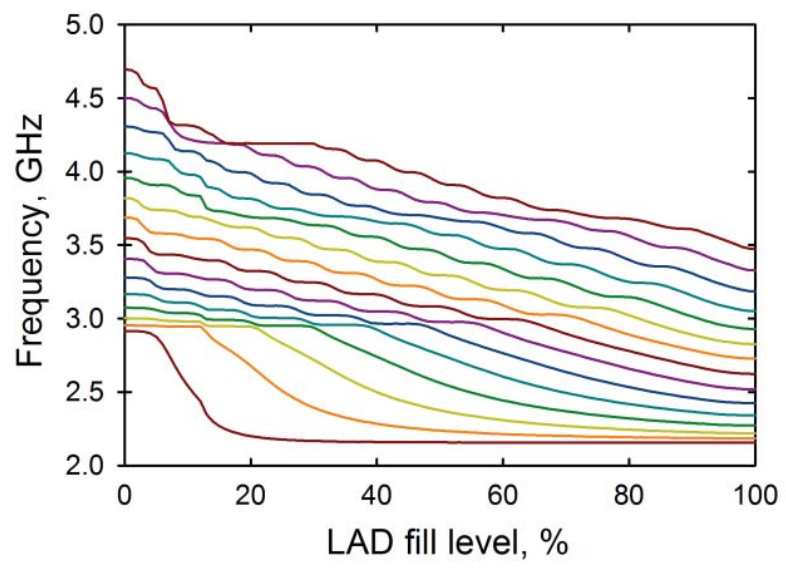

Figure 4. Calculated vertical LAD channel mode frequencies as a function of FC-77 fill level. Different color lines correspond to different RF modes.

The liquid used in these tests, Fluorinert ${ }^{\mathrm{TM}}$ FC-77, was chosen for its good dielectric properties and it also happens to have density and surface tension values close to that of liquid oxygen which makes it a good simulant fluid. The manufacturer's data sheet reports values of $\gamma=0.013 \mathrm{~N} / \mathrm{m}, \rho=1780 \mathrm{~kg} / \mathrm{m} 3$, and a dielectric constant of $\varepsilon$ $=1.9$. We measured the dielectric constant of the liquid in the frequency range $2-4 \mathrm{GHz}$ to be $\varepsilon=1.827$ by measuring the mode frequencies of the LAD channel when it was empty and when full of liquid. The full LAD channel frequencies are related to the empty values by $\left(f_{-} \text {empty } / f_{-} \text {full }\right)^{2}=\varepsilon$.

One of the smaller stainless steel fluid line tubes that was connected to the LAD channel (visible in Figure 1), $7.5 \mathrm{~cm}$ from the end of the channel, was used as an antenna port. A $1.2 \mathrm{~cm}$ long stub antenna was fabricated from a $3.54 \mathrm{~mm}$ diameter, $10 \mathrm{~cm}$ long semi-rigid coax cable by removing $1.2 \mathrm{~cm}$ of the outer conductor at one end of the cable, and attaching a SMA electrical connector at the other end. The antenna was inserted into the LAD via the small fluid line port, and good electrical connection between the outer cable conductor and the fluid line tube was made using conductive copper tape.

After the LAD channel was mounted to the bottom of the open tank, the fluid line and RF connections were made to the LAD and to hermetically sealed fluid and electrical pass-throughs on a flange attached to the upper dome of the tank. A wet-dry sensor was also mounted in the tank at the same level as the top of the LAD screen face, which served as a benchmark level sensor during tank draining. The upper tank dome was then attached to the rest of the tank, and the remaining fluid line connections to a peristaltic pump and fluid container were established, as was the electrical connection of the wet-dry sensor to a simple monitoring circuit and the antenna cable to an RF reflectometer.

When in operation, the RF reflectometer sends a $-10 \mathrm{dBm}$ signal to the antenna and measures the reflected power. In these test, the frequency range of the signal was $2.0-5.4 \mathrm{GHz}$, which was swept using 10,001 discrete points in a three second period. The period between successive RF measurement-sweeps was adjusted from zero seconds for fast measurements during LAD outflow, to once every 3 hours during LAD stability tests.

After establishing a good RF signal, the tank was filled to $95 \%$ fill level, which corresponds to $4.3 \mathrm{~cm}$ above the top end of the LAD channel. The tank was then rotated so that the LAD was horizontal, with the screen channel facing down and fluid ports pointing up. At this point, there were still vapor pockets inside the LAD channel, as evidenced by a changing RF signal depending on liquid position inside the LAD. The fluid pump was turned on, drawing liquid and vapor outside the LAD, which was replenished by liquid flowing through the screen from the tank. The fluid system included a vapor recirculation line to ensure that the tank remained at atmospheric pressure during removal of liquid from the tank. By rocking the tank back and forth during liquid outflow, we found that it was possible to remove the bubbles from the LAD channel. An unchanging RF spectrum was taken as evidence that the air bubbles had been removed from the LAD. Pumping was then stopped, and the tank was rotated back to the 
vertical position. During this LAD filling process, the tank liquid mass dropped by only 2-3\%, which still kept liquid above the LAD when rotated back into the vertical position.

Upon filling the tank and LAD channel, either of two different tests were conducted as part of this study. In the inverted outflow test, referring the reader to Figure 2, fluid was continuously drawn out of the LAD through the upper fluid port while recording RF spectrum data once every three seconds. The tank was drained through the LAD until breakdown occurred, which was noted by both a shift in the RF mode frequencies and bubbles visible in the external fluid line. In the LAD stability test, the tank was drained via the LAD channel bottom fluid port to $20.3 \mathrm{~cm}$ below the top of the $\mathrm{LAD}$, exposing a $14.0 \mathrm{~cm}$ length of screen-channel, and leaving a full liquid column in the LAD channel which was monitored for a period of one month while recording RF spectra once every 3 hours.

When filling or draining the tank, the mass of liquid entering or exiting the tank is measured. A model of the tank is used to convert the measured mass of liquid inside the tank to a percentage fill by volume. The model is also used to convert liquid mass to height of liquid in the tank. The model is considered accurate to $\pm 0.2 \%$ by volume, and we note that the benchmark wet-dry sensor which was mounted parallel to the face of the tank dome flange indicated a fill level (based on the mass of liquid in the tank at the point it changed from wet to dry) of $80.6 \%$, in excellent agreement with the model value of $80.6 \%$ fill at the flange location.

\section{Results and Discussion}

The inverted outflow test is a convenient way to characterize LAD breakdown during flow conditions ${ }^{12}$, and can give a measure of the liquid column height supported by the LAD when breakdown occurs. From Eq. (2), we expect the LAD to hold a $21.3 \mathrm{~cm}$ column of FC-77 liquid before breakdown. The screen-face of the LAD used in these tests was $48.3 \mathrm{~cm}$ long, centered on the $61.0 \mathrm{~cm}$ LAD channel, leaving a $6.4 \mathrm{~cm}$ length region at each end that could hold additional liquid (or trapped air) but was not affected by the screen bubble-point. Thus, the LAD was predicted to hold up to a $27.7 \mathrm{~cm}$ total column of liquid above the tank bulk fluid fill level. During the inverted outflow test, conducted at a flow rate of $1.33 \mathrm{~kg} / \mathrm{min}$, the RF data were recorded continuously, visual observations of the RF spectra were made to observe frequency shifts of the modes, and the external fluid lines were observed for evidence of bubbles in the outflow.

Figure 5 shows a plot of the measured LAD channel mode frequencies as a function of time during the inverted outflow test. Visual observation of bubbles occurred in the fluid lines at approximately 15:48:00, three minutes after observing shifts in the mode frequencies. The delay is not surprising given that the fluid port used for outflow resides below the top of the LAD channel. The gauged LAD \%-fill versus time plot, also shown in Fig. 5, shows that the RFMG gauged fill level of the LAD began decreasing at nearly the same time as visual observations of the change in mode frequencies. Note that the gauged output, having $1 \%$ output resolution in these tests, indicates that the LAD was not completely full at the start of the test. It was noted during this test that removal of all the air from
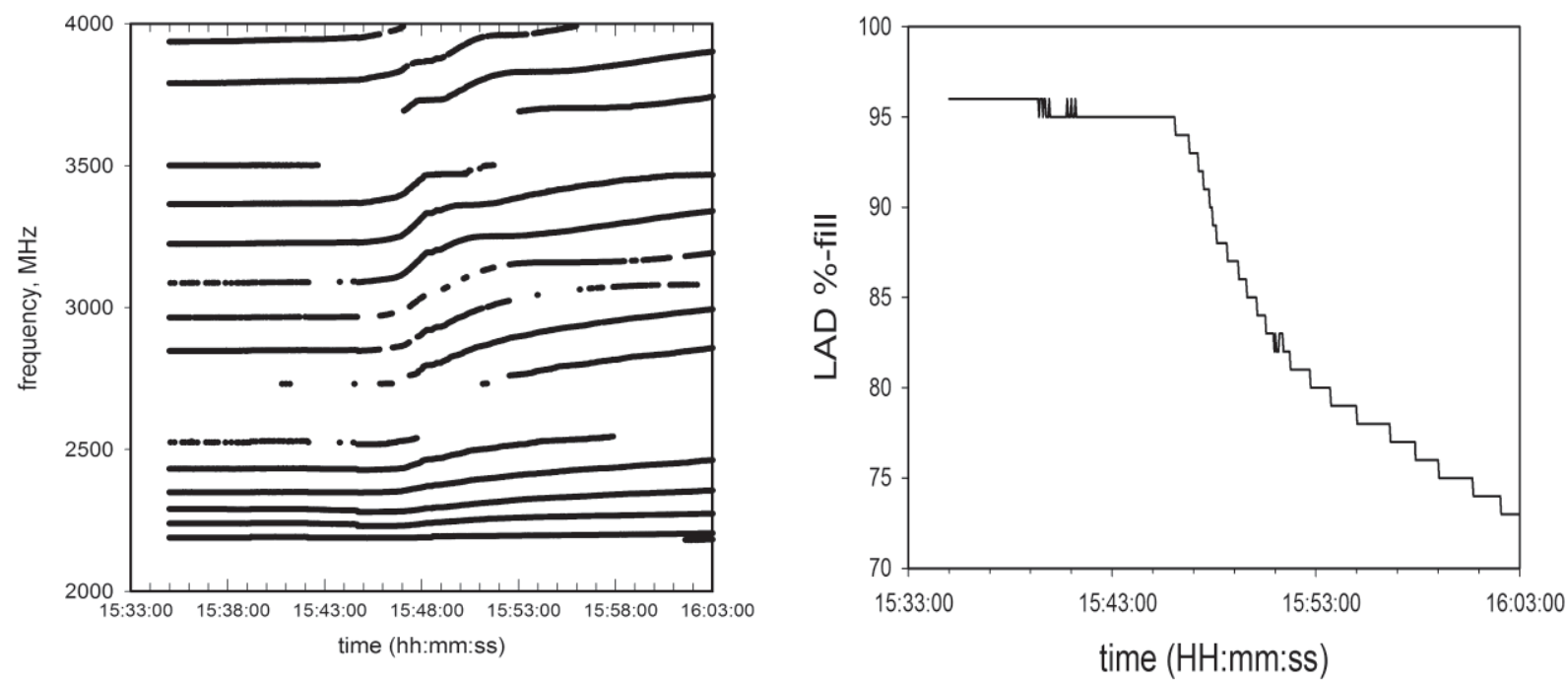

Figure 5. Inverted outflow test results: (left) Detected mode frequencies as a function of time, and (right) LAD fill level as a function of time as gauged by the RFMG. 
the LAD channel during filling was not completely successful. Nevertheless, the bubble resided in the upper portion of the LAD, above the screened face area which extends up to the $90 \%$ LAD fill level.

At the time that the breakdown occurred and vapor was ingested into the LAD channel, the tank liquid level was $25.9 \mathrm{~cm}$ below the top of the LAD channel. Thus, the measured fluid height at breakdown is in good agreement with the predicted value of $27.7 \mathrm{~cm}$. The results demonstrate that the RF sensor can provide a relatively simple method for detecting breakdown in a LAD.

In a long term stability test, also demonstrating the utility of the RF sensor in the LAD, the tank and LAD was filled with FC-77, then drained through the bottom fluid port of the LAD (the top port was capped) until the tank liquid level was $20.3 \mathrm{~cm}$ below the top of the LAD channel, resulting in an exposed screen-channel length of $14.0 \mathrm{~cm}$. The stability of the column of liquid in the LAD channel was monitored by the RF sensor once every 3 hours for a period of one month. The data system recorded the RF spectra, and was post-processed to convert the measured frequencies to a $\mathrm{LAD} \%$-fill value as a function of time. The results are shown in Figure 6. The plot shows that the liquid level in the LAD steadily decreased by $-0.5 \%$ /day during the first three weeks, with a noticeable change in slope around day 21, followed by breakdown, or loss of the liquid column, on day 26.

A reasonable explanation for the change in slope on day 21 is that the liquid column height had decreased to the level of the screen, which is $6.4 \mathrm{~cm}$

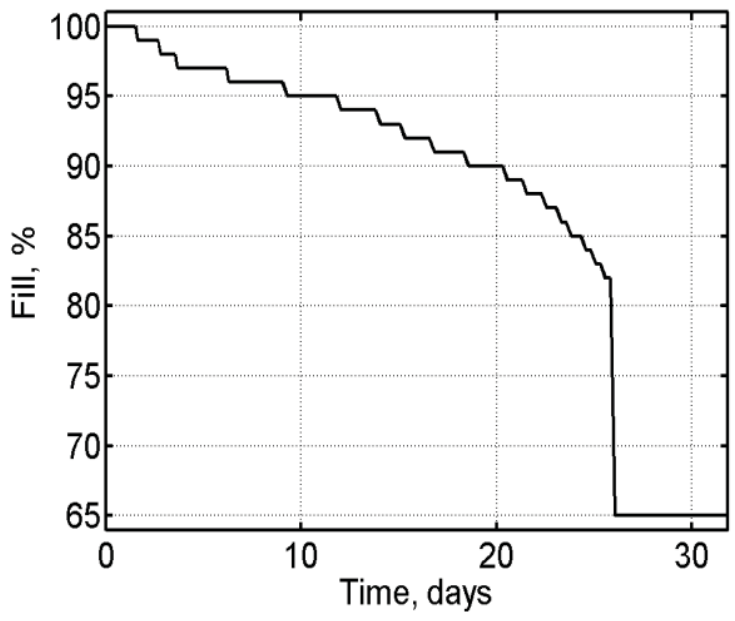

Figure 6. RF gauged fill level in the LAD as a function of time during the long-term stability test. or $10.4 \%$ below the top of the LAD channel, thus exposing the LAD screen to vapor on both sides of the screen. The initial, steady decrease of liquid height in the LAD channel during the stability test is likely due to a combination of air diffusion in the FC-77, which would accumulate as a growing vapor pocket in the top of the channel, and liquidloss via evaporation from the screen-channel face back into the bulk liquid reservoir. Although the vapor is in a saturated state at the tank liquid-vapor interface, there is a gravity induced pressure gradient in the vapor phase that causes the partial pressure of the FC-77 to decrease as a function of height. The loss of liquid by evaporation from the screen face would be partially compensated for by wicking of liquid up the screen and LAD channel from the bulk reservoir. It is possible that the balance between air diffusion, liquid evaporation from the screen, and liquid mass transport up the LAD channel reached a breaking point at the $82 \%$ LAD channel fill level, when a $4.6 \mathrm{~cm}$ long section of screen-channel face was exposed to air on both sides of the screen. Although such a breakdown process may not happen in a microgravity condition, it does demonstrate the utility of the RF sensor for investigating fundamental characteristics of screen-channel LAD's in a $1 \mathrm{~g}$ environment. Previously reported measurements of screen-channel LAD stability in a $1 \mathrm{~g}$ environment are limited to 10 minutes during stepped expulsion testing ${ }^{12}$. As a suggestion for further work in this area, it would be valuable to create a model of the LAD stability process, and conduct a similar LAD stability test with the RF sensor using a liquid cryogen and compare the results against the model predictions.

\section{Conclusions}

The RF mass gauge sensor has been used to monitor the liquid level in a screen-channel liquid acquisition device during inverted outflow and long-term LAD stability tests. It was shown that the sensor can detect breakdown in the LAD channel, and can measure changes in LAD fill over time during stability tests. The antenna sensor in the LAD channel is relatively non-intrusive, comprised of a simple monopole stub antenna, and is capable of detecting vapor pockets anywhere along the LAD channel. Although the lower limits of vapor pocket size were not investigated here, it is reasonable to expect the sensor to detect a change in $0.1 \%$ of fill level by monitoring several of the higher mode frequencies. 


\section{Acknowledgments}

This work was supported by NASA, through the Space Technology Mission Directorate Cryogenic Propellant Storage and Transfer Project. The authors wish to thank Jason Hartwig and David Chato for useful discussions and providing the LAD hardware used in this test.

\section{References}

1. http://www.nasa.gov/offices/oct/strategic_integration/technology_roadmap.html

2. National Research Council. NASA Space Technology Roadmaps and Priorities: Restoring NASA's Technological Edge and Paving the Way for a New Era in Space. Washington, DC: The National Academies Press, 2012.

3. Rollins, J. R., Grove, R. K., and Jaekle, D. E., Jr., "Twenty-Three Years of Surface Tension Propellant Management System Design, Development, Manufacture, Test, and Operation”, AIAA-85-1199, 1985.

4. Shirron, P.J., DiPirro, M.J., and Tuttle, J., "Flight performance of the SHOOT liquid acquisition devices", Cryogenics, Vol. 34, pp 361-368 (1994).

5. Jaekle Jr., D. Propellant Management Device Conceptual Design and Analysis: Galleries; AIAA-97-2811; AIAA/SAE/ASME/ASEE 33rd Joint Propulsion Conference; 1997; Seattle, WA.

6. Chato, D.J. and Kudlac, M.T., Screen Channel Liquid Acquisition Devices for Cryogenic Propellants; AIAA-20023983; 38th Joint Propulsion Conference and Exhibit cosponsored by the AIAA, ASME, SAE, and ASEE, Indianapolis, IN, 2002.

7. Zimmerli, G.A., Asipauskas, M., Wagner, J.D., and Follo, J.C., "Propellant Quantity Gauging Using the Radio Frequency Mass Gauge", AIAA 2011-1320, 49th AIAA Aerospace Sciences Meeting, January 2011, Orlando, FL.

8. Zimmerli, G.A., (2013), U.S. patent no. 8353209 B1. Washington, DC: U.S. Patent and Trademark Office.

9. In order to describe procedures adequately, it is occasionally necessary to identify commercial products by the manufacturer's name or label. In no instance does such identification imply endorsement by NASA, nor does it imply that the particular product is necessarily the best available for the purpose.

10. Hartwig, J., McQuillen, J., and Jurns, J., "Screen Channel LAD Bubble Point Tests in Liquid Oxygen", Journal of Thermophysics and Heat Transfer (accepted for publication), 2014.

11. COMSOL Multiphysics, Version 3.5a, COMSOL, Inc., 1 New England Executive Park, Suite 350, Burlington, MA 01803, USA.

12. Meserole, J.S. and Jones, O.S., "Pressurant Effects on Cryogenic Liquid Acquisition Devices", Journal of Spacecraft and Rockets Vol. 30, pp. 236-243 (1993). 